\title{
Improving Healthcare Delivery Through Patient Informatics and Quality Centric Data
}

\author{
Bruce I. Reiner
}

Published online: 28 January 2011

(C) Society for Imaging Informatics in Medicine 2011

Over the past two decades, medical practice has become transformed by the digitization of medical data. This phenomenon was facilitated by the creation, refinement, and integration of medical information system technologies, which in turn led to the creation of medical informatics, which has been defined as "the field that concerns itself with the cognitive, information processing, and communication tasks of medical practice, education, and research [1].

The digitization of medical data has led to a number of dramatic changes in healthcare deliverables and expectation. In radiology, the ability to instantaneously access medical imaging data from essentially any location led to heightened expectations in operational efficiency (e.g., report turnaround times), decreased face to face physician-radiologist consultations, and increased provider sub-specialization [2-4]. The traditional geographic barriers which protected service providers (e.g., radiologists) began to dissolve and provided consumers with increased options through teleradiology and portable electronic media.

These changes in service expectations fostered a new wave of technology innovation in radiology aimed at improving quality, efficiency, and accuracy. Computerized scheduling and digital dashboards became integrated into administrative workflow, providing more timely service and the ability to monitor departmental workflow in real-time.

\section{B. I. Reiner}

Department of Radiology,

Veterans Affairs Maryland Healthcare System,

10 North Greene Street,

Baltimore, MD 21201, USA

B. I. Reiner $(\bowtie)$

11402 Newport Bay Drive,

Berlin, MD 21811, USA

e-mail: breiner1@comcast.net
Speech recognition technologies were created to improve report turnaround, while structured reporting technologies attempted to address long-standing deficiencies in report clarity and organization. Computerized decision support technologies, such as computer-aided detection software, provided a method for improving diagnostic accuracy and radiologist confidence.

These initiatives and technology developments have led to improvements in data accessibility, operational efficiency, decision making, and education among medical service providers. At the same time, ongoing efforts are being made to standardize medical data collection, leading to the creation of referenceable databases, which can be used in the creation of "best practice" guidelines and adoption of evidence-based medicine.

While these provider-oriented initiatives and technology developments were taking place, a number of economic, sociologic, and technology movements were afoot within the population of healthcare consumers (i.e., patients). Patients were being asked to take on greater personal responsibility for healthcare costs, through increased insurance deductibles and co-pays, making them more cognizant of service costs. The traditional paternalistic physician-patient relationship was being questioned, with patients demanding and expecting a greater voice in their own healthcare decision making. The Internet created easy access to personalized healthcare information through the creation of healthcare portals, electronic scorecards, and electronic patient records. The combination of these factors led to a new era of patient empowerment which has arguably disrupted the status quo in the traditional provider-patient relationship.

While medical informatics has traditionally served as an educational tool for healthcare providers, it is by no means limited to this sector and can be easily adapted to the specific 
needs and preferences of consumers. Consumer health informatics has been defined as the branch of medical informatics that analyzes consumers' needs for medical information, studies and implements methods of making information accessible to consumers, and models and integrates consumers' preferences into medical information systems [5]. In addition to creating a better informed patient population, consumer health informatics provides the potential to improve clinical outcomes, strengthens the providerpatient relationship, and improves utilization of health service resources $[6,7]$.

One potential starting point for such an endeavor would be the creation of standardized databases, which could present patients with objective and reproducible data to assist in service provider selection. The data could record and analyze measures of quality, safety, cost-efficacy, operational efficiency, and customer satisfaction and be customizable to individual patient preferences and medical problems. The proposed interactive system could allow patients to prioritize the metrics of greatest importance while taking into account unique patient attributes such as geographic location, insurance profile, and health status. The database would analyze these input criteria and provide the patient with a list of prospective service providers in rank order, which fulfills the search criteria of interest. The methodology for creating such a database has been described [8-11], with data collection, analysis, and validation performed by neutral, non-profit third parties with expertise in healthcare service.

While this may be perceived as threatening to the service provider community, it is arguably empowering and liberating. Physicians who engage in self-referrals or quid pro quo arrangements could now be evaluated in a neutral and unbiased fashion based upon objective performance data. Providers who have utilized substandard technology and engaged in lax quality assurance practice would now be scrutinized in accordance with comparable peer statistics. A third party payer who directs patients to a specific service provider based upon pre-negotiated contracts would have to justify provider selection, in keeping with quality and safety performance data. Providers who proactively invest in quality and safety would be recognized for their efforts, while those providers with deficient ratings would have the opportunity to utilize the data for internal quality improvement.

Creating objective informational resources for educating and informing healthcare consumers is a logical extension to ongoing clinical and technical initiatives. Like it or not, service providers should embrace the opportunity to be evaluated in a fair, objective, and reproducible fashion. An educated and informed consumer population has the potential to enhance clinical outcomes, and this should always be the primary goal of healthcare service providers. Success in healthcare delivery should be predicated on survival of the fittest, not survival of the cheapest.

\section{References}

1. Greenes RA, Shortliffe EH: Medical informatics: an emerging academic discipline and institutional priority. JAMA 263:11141120,1990

2. Reiner B, Siegel E, Protopapas Z, et al: Impact of filmless radiology on the frequency of clinician consultations with radiologists. AJR 173:1169-1172, 1999

3. Twair AA, Torreggiani WC, Mahmud SM, et al: Significant savings in radiologic report turnaround time after implementation of a complete picture archiving and communication system. J Digit Imaging 13:175-177, 2000

4. Bradley WG: Off-site teleradiology: the pros. Radiology 248:337341, 2008

5. Eysenbach G: Consumer health informatics. BMJ 320:17131716,2000

6. Kassirer JP: Patients, physicians, and the internet. Health Aff 19:115-123, 2000

7. Murray E, Lo B, Pollack L, et al: The impact of health information on the Internet on the physician-patient relationship. Arch Intern Med 163:1727-1734, 2003

8. Reiner BI: Quantifying radiation safety and quality in medical imaging. Part I: creating the infrastructure. J Am Coll Radiol 8:558-561, 2009

9. Reiner BI: Quantifying radiation safety and quality in medical imaging. Part II: the radiation scorecard. J Am Coll Radiol 9:615619, 2009

10. Reiner BI: Quantifying radiation safety and quality in medical imaging. Part III: the quality assurance scorecard. J Am Coll Radiol 10:694-700, 2009

11. Reiner BI: Quantifying radiation safety and quality in medical imaging. Part IV: the medical imaging agent scorecard. J Am Coll Radiol 2:120-124, 2010 